九州大学学術情報リポジトリ

Kyushu University Institutional Repository

\title{
Multiple schwannomas in the oral floor: Case report
}

\section{Kubota, Yasutaka}

Department of Oral and Maxillofacial Surgery, Graduate School of Dental Science, Kyushu University

\section{Yanai, Yuta}

Department of Oral and Maxillofacial Surgery, Graduate School of Dental Science, Kyushu University

\section{Kumamaru, Wataru}

Department of Oral and Maxillofacial Surgery, Graduate School of Dental Science, Kyushu University

Mori, Yoshihide

Department of Oral and Maxillofacial Surgery, Graduate School of Dental Science, Kyushu University

http://hdl. handle. net/2324/25689

出版情報: British Journal of Oral and Maxillofacial Surgery. 49 (6), pp. e33-e35, 2011-09. Elsevier B.V.

バージョン:

権利関係: (C) 2010 British Association of Oral and Maxillofacial Surgeons 
Multiple schwannomas in the oral floor: A case report

Yasutaka Kubota*, Yuta Yanai, Wataru Kumamaru, Yoshihide Mori

Department of Oral and Maxillofacial Surgery, Graduate School of Dental Science, Kyushu University, 3-1-1 Maidashi, Higashi-ku, Fukuoka 812-8582, Japan.

*Address correspondence and reprint requests to: Yasutaka Kubota, DDS, PhD. Department of Oral and Maxillofacial Surgery, Graduate School of Dental Science, Kyushu University, 3-1-1 Maidashi, Higashi-ku, Fukuoka 812-8582, Japan.

FAX: +81-92-642-6392, e-mail: yasu@dent.kyushu-u.ac.jp 


\begin{abstract}
We herein present a rare case of multiple schwannomas in the oral floor that occurred in a 62-year-old male patient. No café-au-lait spots on the skin, cutaneous tumors, the eighth nerve tumors, or lens opacity was detected. There was no history of neurofibromatosis 2 or definite schwannomatosis in any first-degree relative. A CT scan and MR imaging demonstrated the presence of two isolated and well-bordered tumors in the oral floor. Both tumors were histopathologically diagnosed as schwannoma. Immunohistochemically, the tumor cells were positive for the S-100 protein and CD34, while the cells were negative for epithelial membrane antigen (EMA) and Factor XIIIa. This case, therefore, met the diagnostic criteria for schwannomatosis.
\end{abstract}

Keywords: multiple shwannomas; oral floor; schwannomatosis

\title{
Introduction
}

Schwannoma is a relatively uncommon benign nerve sheath tumor in the oral cavity and usually occurs as a solitary tumor. ${ }^{1,2}$ Multiple schwannomas are often detected in neurofibromatosis (NF) 2 which is an autosomal dominantly inherited disorder. ${ }^{3}$ Recently, schwannomatosis is recognized as the third major form of $\mathrm{NF}^{4}$ Schwannomatosis is characterized by multiple non-intradermal or non-vestibular schwannomas. The presence of multiple schwannomas in a single patient thus suggests a possible association with NF2 or schwannomatosis. In this paper, we present a rare case 
of multiple schwannomas in the oral floor which met the diagnostic criteria for schwannomatosis.

\section{Case report}

A 62-year-old male was referred to Kyushu University Hospital for a painless swelling of the oral floor on the left side in May 2009. The patient had noticed the swelling about 3 months prior to visiting the hospital. Clinical examination revealed the presence of a tumor which was well-bordered, non-tender, and non-compressible. The overlying oral mucosa of the tumor was normal (Figure 1). No café-au-lait spots on the skin, cutaneous tumors, the eighth nerve tumors, or lens opacity was detected. There was no history of NF2 or definite schwannomatosis in any first-degree relative.

Computed tomography (CT) examination demonstrated the presence of large $(40 \times 30$ $\mathrm{mm})$ and small $(10 \times 10 \mathrm{~mm})$ low-density tumors in the oral floor. Both tumors were well-bordered and no communication was detected between the two tumors. T1-weighted magnetic resonance (MR) image showed that both tumors were homogenously hypointensive, while T2-weighted MR image and Gadolinium-enhanced T1-weighted MR image showed that both tumors were heterogeneously hyperintensive (Figure 2). No vestibular tumor was detected.

Schwannoma or neurofibroma was suggested by an incisional biopsy of the large tumor. The large and small tumors were completely enucleated through an intraoral approach under general anaesthesia. Both tumors were encapsulated and were completely distinguished from each other. There was no apparent communication 
between the tumors and nerve bundles. Postoperative healing was uneventful.

Histopathologically, the specimens of the large and small tumors revealed a characteristic encapsulated schwannoma composed of Antoni-A and Antoni-B patterns (Figure 3). Immunohistochemical staining revealed that the tumor cells were positive for the S-100 protein but negative for epithelial membrane antigen (EMA) and Factor XIIIa. The tumor cells in the Antoni-B region were positive for CD34.

\section{Discussion}

We herein present a rare case of multiple schwannomas in the oral floor. Histopathologically, the Antoni-B structure of schwannoma is sometimes difficult to distinguish from neurofibroma. Recently, nerve sheath tumors with hybrid features of neurofibromas and schwannomas have been reported in the literature. ${ }^{5}$ Immunohistochemically, schwannomas are positive for S-100 protein and CD34, but negative for Factor XIIIa. ${ }^{6-9,10}$ On the other hand, neurofibromas are positive for S-100 protein, CD34, and Factor XIIIa. ${ }^{6,8,9}$ Therefore, the staining intensities for Factor XIIIa are clearly different between schwannoma and neurofibroma. The tumor cells in our case were negative for Factor XIIIa, supporting the diagnosis of the tumors in this case as being schwannomas.

The diagnostic criteria for schwannomatosis consist of a two-tiered paradigm for "definite" and "possible" schwannomatosis (Table 1). Possible schwannomatosis is permitted when a patients is older than 45 years of age, does not have symptoms of an eighth nerve dysfunction, and the tumors are multiple non-intradermal schwannomas. 
Therefore, our case may meet the diagnostic criteria for possible schwannomatosis.

A surgical excision is the treatment of choice for schwannoma. Malignant change is extremely rare in a benign schwannoma and no reports of such change occurring in the cases of multiple schwannomas. In our case, no recurrence of the tumor was observed during the 1-year follow-up period.

\section{Acknowledgments}

The authors thank Dr T. Chikui of Department of Oral Radiology, Graduate School of Dental Science, Kyushu University for his pertinent advice.

\section{Conflict of interest}

The authors do not have any financial and personal relationships with other people or organizations that inappropriately influence this work. 


\section{References}

1. Ducic Y. Schwannoma of the floor of the mouth. Otolaryngol 2003; 129: 144-6.

2. Kawakami R, Kaneko T, Kadoya M, Matsushita T, Fujinaga Y, Oguchi K, Kurashina K. Schwannoma in the sublingual space. Dentomaxillofac Radiol 2004; 33: 259-61.

3. Ferner RE. Neurofibromatosis 1 and neurofibromatosis 2: a twenty first century perspective. Lancet Neurol 2007; 6: 340-51.

4. Baser ME, Friedman JM, Evans DG. Increasing the specifity of diagnostic criteria for schwannomatosis. Neurology 2006; 66: 730-2.

5. Feany MB, Anthony DC, Fletcher CDM. Nerve sheath tumours with hybrid features of neurofibroma and schwannoma: a conceptual challenge. Histopathology 1998; 32: 405-10.

6. Chrysomali E, Papanicolaou SI, Dekker NP, Regezi JA. Benign neural tumors of the oral cavity: a comparative immunohistochemical study. Oral Surg Oral Med Oral Pathol Oral Radiol Endod 1997; 84: 381-90.

7. Hirose T, Sano T, Hizawa K. Ultrastructural localization of S-100 protein in neurofibroma. Act Neuropathol 1986; 69: 103-10.

8. Weiss SW, Nickoloff BJ. CD-34 is expressed by a distinctive cell population in peripheral nerve, nerve sheath tumors, and related lesions. Am J Surg Pathol 1993; 17: 1039-45.

9. Hirose T, Tani T, Shimada T, Ishizawa K, Shimada S, Sano T. Immunohistochemical demonstration of EMA/Glut1-positive perineurial cells and CD34-positive fibroblastic cells in peripheral nerve sheath tumors. Mod Pathol 2003; 16: 293-8. 
10. Theaker JM, Gatter KC, Puddle J. Epithelial membrane antigen expression by the perineurium of peripheral nerve and in peripheral nerve tumours. Histopathology 1988; 13: 171-9.

\section{Legends}

Figure 1. Intraoral view. A large tumor that presented in the oral floor on the left side was well-bordered, firm, and non-compressible.

Figure 2. MR image. MR image demonstrated the presence of well-bordered hyperintensive large and small tumors (white arrows) in the oral floor. The small tumor was located inferior to the large one.

Figure 3. Histopathological finding of the large tumor. Hematoxylin-eosin (HE) stain in Antoni-A region composed of interwoven bundles of long bipolar spindle cells (100X magnification). 
Fig. 1

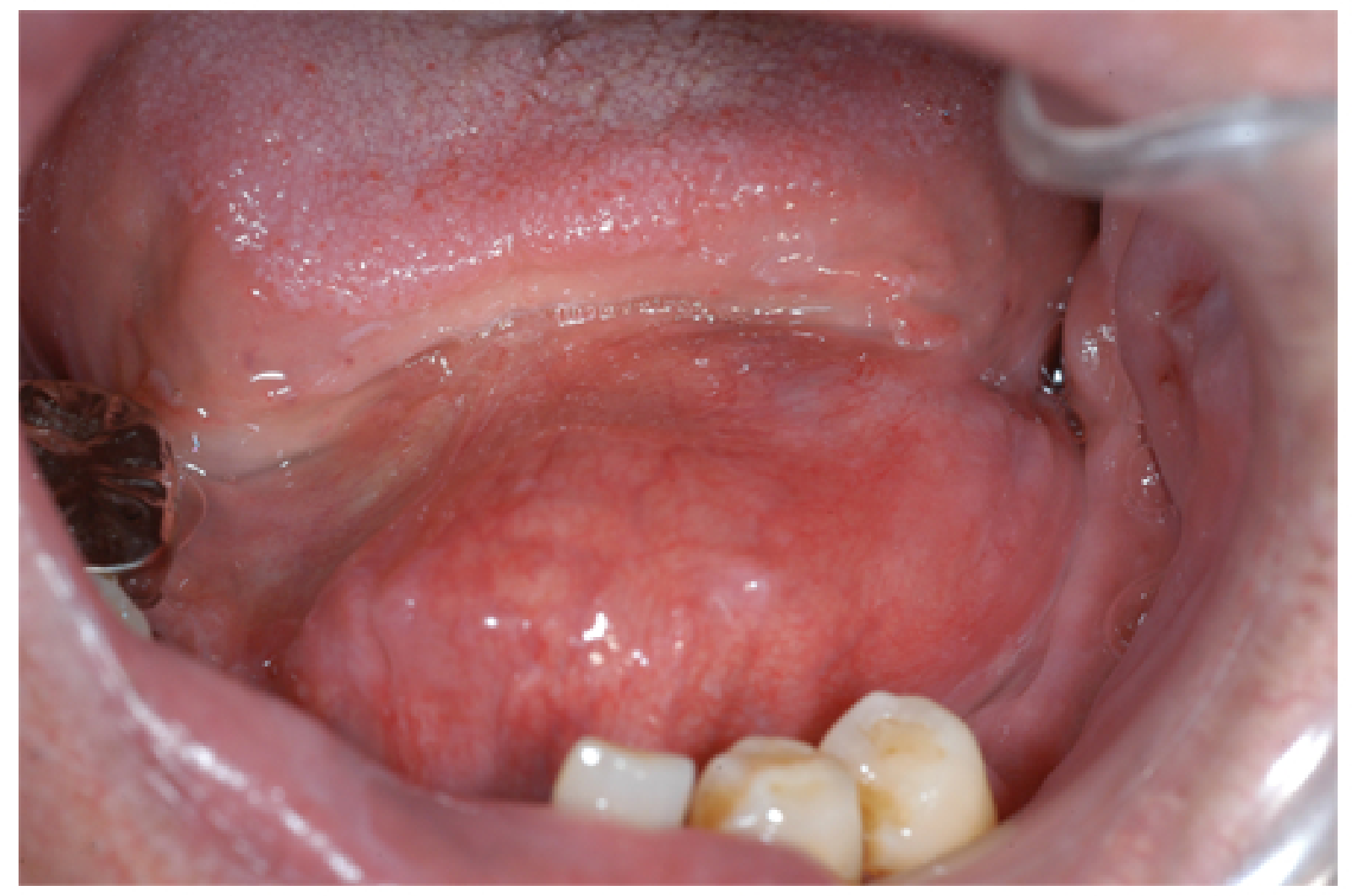


Fig. 2

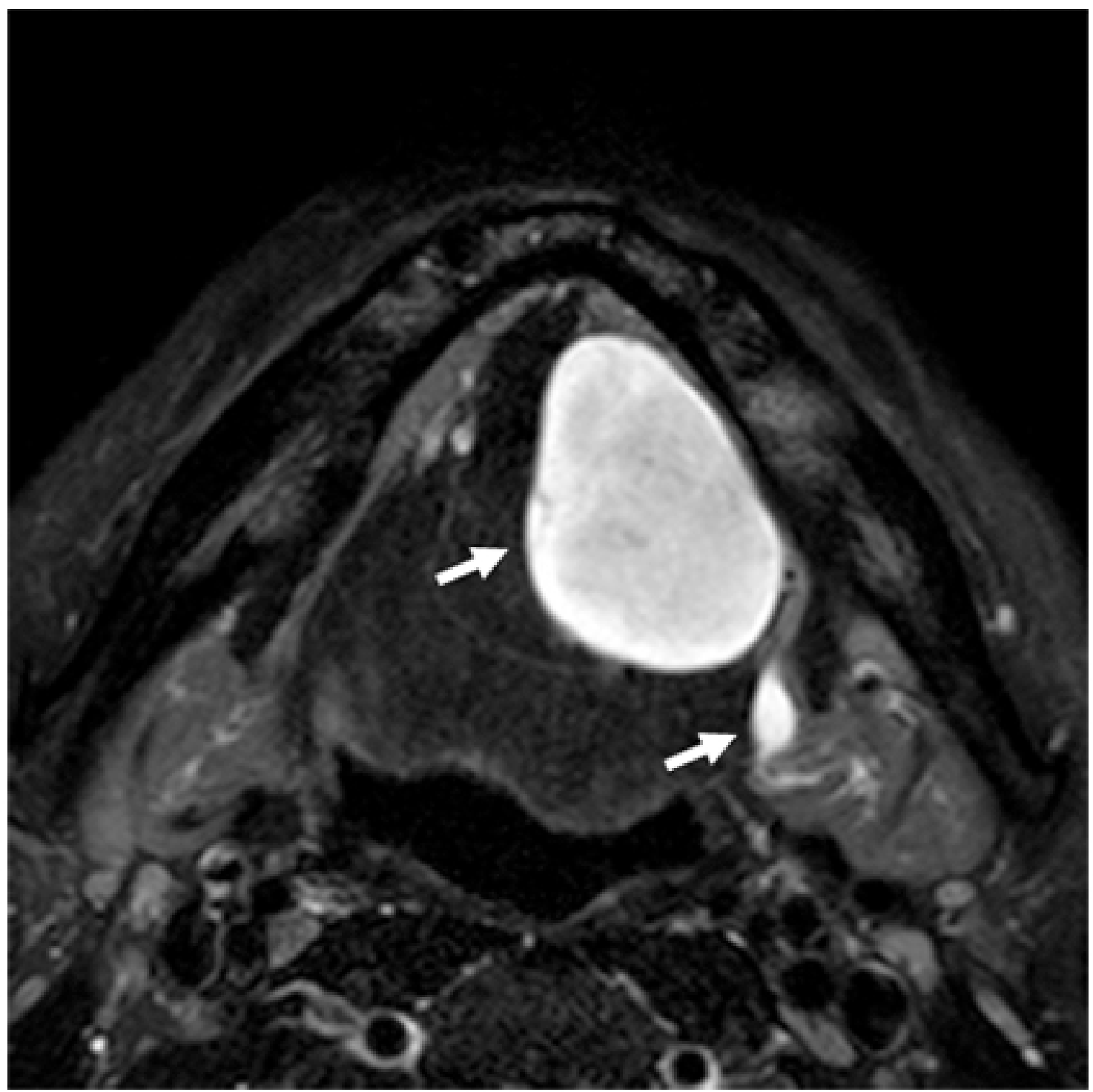




\section{Fig.3}

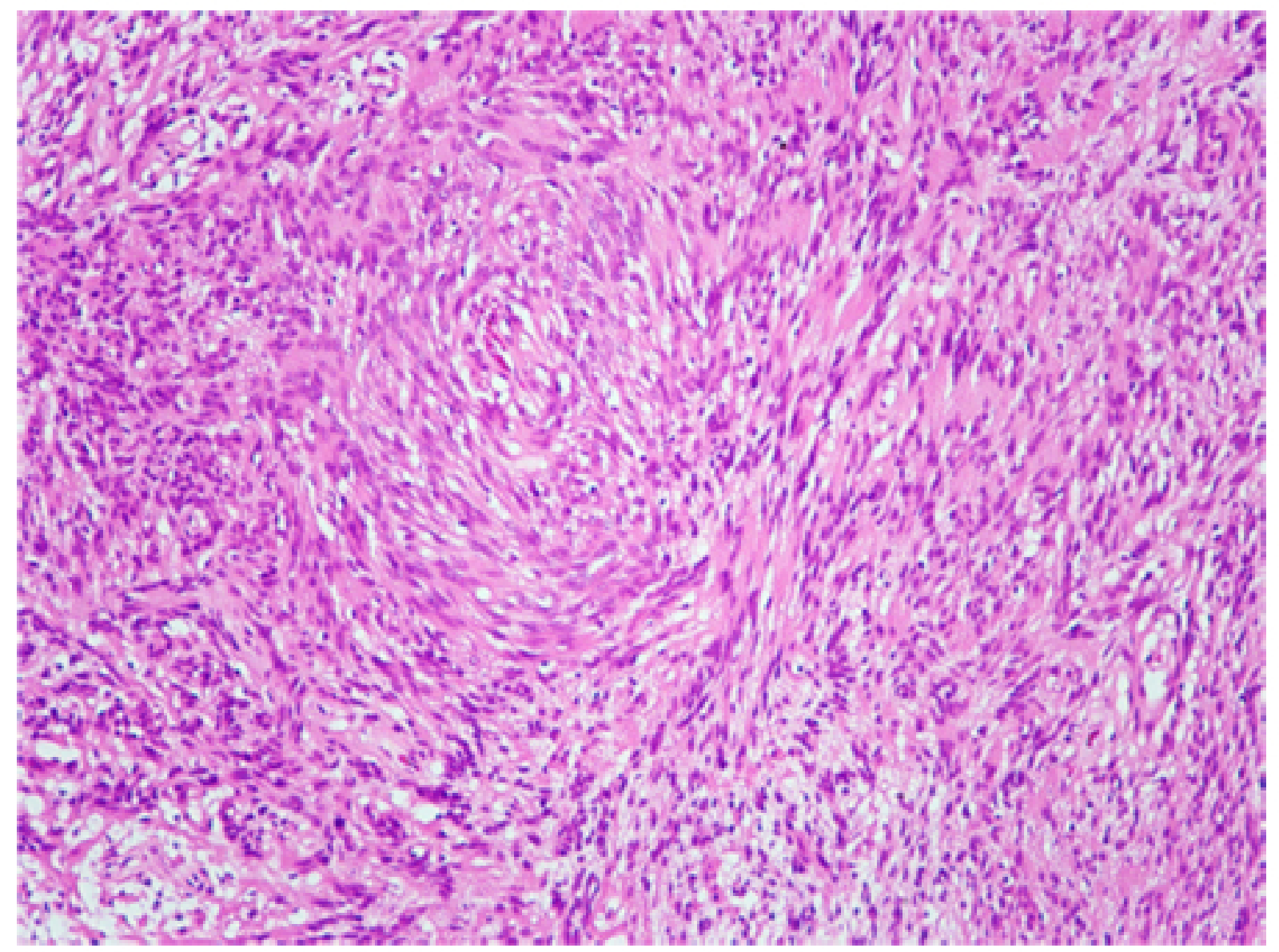


Table 1. Diagnostic criteria for NF1, NF2, and schwannomatosis

I. NF1 (NIH criteria ${ }^{3}$ : 1987); autosomal dominantly inherited disorder (chromosome 17q)

Two or more of the below criteria

i) Café-au-lait spots (6 or more) ( $>5 \mathrm{~mm}$ in children or $>15 \mathrm{~mm}$ in adults)

ii) Cutaneous or subcutaneous neurofibromas ( 2 or more) or one plexiform neurofibroma

iii) Axillary or groin freckling

iv) Optic glioma

v) Lisch nodules (2 or more)

vi) Bone lesion with sphenoid wing dysplasia or bowing of the long bones with/without pseudoarthrosis

vii) First-degree relative with NF1

II. NF2 (Manchester criteria ${ }^{3}$ : 1992); autosomal dominantly inherited disorder (chromosome 22q)

1) Bilateral vestibular schwannomas

2) First-degree relative with NF2 and unilateral vestibular schwannoma or two of meningioma, schwannoma, glioma, neurofibroma, posterior subcapsular lens opacity

3) Unilateral vestibular schwannoma and two of meningioma, schwannoma, glioma, neurofibroma, posterior subcapsular lens opacity

4) Multiple meningiomas ( 2 or more) and unilateral vestibular schwannoma or two of schwannoma, glioma, neurofibroma, cataract

III. Schwannomatosis (Baser et al. $\left.{ }^{4}: 2006\right)$

(1) Definite schwannomatosis

1) Age $>30$ years and non-intradermal schwannomas ( 2 or more)

2) One pathologically confirmed schwannoma and a first-degree relative who meets the above criteria

(2) Possible schwannomatosis

1) Age $<30$ years and non-intradermal schwannomas (2 or more)

2) Age $>45$ years and no symptoms of eighth nerve dysfunction and non-intradermal schwannomas (2 or more)

3) Radiographic evidence of a schwannoma and a first-degree relative with definite schwannomatosis 